\title{
Compound palmar ganglion associated with tuberculous arthritis of wrist causing compression of the median and ulnar nerves: Management
}

\author{
Kosalaraman Padmanabhan ${ }^{1, *}$, Balamurugan Palanisamy ${ }^{2}$, Mohan Prasad Muthusamy ${ }^{3}$ \\ ${ }^{\mathbf{1}}$ Associate Professor, ${ }^{2}$ Senior Assistant Professor, ${ }^{3}$ Junior Resident, Dept. of Orthopaedics, Coimbatore Medical College,
} Coimbatore, Tamil Nadu, India

*Corresponding Author:

Email: pkrvidya.2002@gmail.com

\begin{abstract}
50 years old male, agricultural labourer, presented with 5 months history of pain and swelling of the right wrist and hand. There was no history of any previous trauma or other constitutional symptoms. Patient had associated numbness of fingers of right hand with reduced hand grip. On clinical examination, a dumb bell shaped swelling was noted on the palmar aspect of wrist and hand with cross fluctuation positive. Blood investigations revealed elevated ESR and CRP. X ray right wrist showed features of early arthritis. Nerve conduction study showed features of entrapment neuropathy of median and ulnar nerve. MRI wrist showed features of tuberculous tenosynovitis of flexor tendons with early arthritis of right wrist. Patient was treated by excision biopsy of the compound palmar ganglion and started on anti-tuberculous drugs after histopathology confirmed tuberculous tenosynovitis. Post operatively the patient improved clinically with reduction in pain and on follow-up numbness and functional activity of right hand improved well.
\end{abstract}

Keywords: Compound palmar ganglion, Tuberculous tenosynovitis, Tuberculous arthritis of the wrist, Melon seed body, Rice body.

\section{Introduction}

Compound palmar ganglion is a rare clinical presentation of the tuberculous arthritis of wrist and hand. It is predominantly seen in males and usually involves the dominant limb. Usually it presents with symptoms of carpal tunnel syndrome due to median nerve compression. Combined sensorimotor polyneuropathy affecting both median and ulnar nerves is a rare presentation. Early diagnosis and excision biopsy has to be done to prevent subsequent bone involvement as well as permanent neurological disability. It responds well to early excision and antituberculous treatment with near normal functional recovery.

\section{Case History}

A 50 years old male, agricultural labourer by occupation came to the orthopaedic outpatient clinic, with painful swelling of the right wrist for past 5 months. He had history of injury to the right middle finger 10 months back for which he did not take any treatment resulting in persistent deformity of the finger. The patient gave history of haemoptysis two years back for which surgical procedure done on the right side of chest, the details of which could not be traced out. Swelling was insidious in onset, started in wrist and gradually progressed to the hand. Pain developed two months after swelling, insidious onset, dull aching type, aggravated by work and relieved with rest. There was history of night cries. The patient had difficulty in activities of daily living using his right hand for holding objects and for feeding. Patient had significant weight loss. There was no past history of contact with proven case of tuberculosis, rheumatoid arthritis and any cervical spine pathology. Patient was not a known case of diabetic or hypertensive.

On clinical examination, there was a $8 \times 4 \mathrm{~cm}$ diffuse swelling on the flexor aspect of wrist and hand extending above and below the flexor retinaculum (Fig. 1 and 2). There was mallet finger deformity of middle finger due to old trauma. The skin over the swelling appeared normal .The swelling was cystic and crossfluctuation was positive. There was no warmth. The swelling became more prominent on flexing the wrist against resistance indicating the plane of the swelling. The bony landmarks were normal on palpation except for the wrist joint line tenderness dorsally. The passive range of movements of right wrist was painful and restricted. Transillumination test was negative. Clinical examination of the elbow, shoulder and cervical spine appeared to be normal. Radial pulse was felt equally on both sides. There were no regional lymph nodes palpable. Neurological examination revealed decreased sensation in the territory of median and ulnar nerve with the radial nerve unaffected. The motor functions of the median and ulnar nerves were found to be intact.

On investigation, there was elevated ESR, CRP and lymphocyte count. Chest radiograph was normal and sputum was negative for AFB. Mantoux test was positive. X ray right wrist (Fig. 3) showed features of juxta-articular osteoporosis with irregular narrowing of radiolunate and distal radioulnar joint space with soft tissue shadow on the flexor aspect of wrist and hand. Ultrasonogram of the wrist and hand revealed irregular thickening of synovial sheath with peri-tendinous fluid collection suggestive of tenosynovitis probably of 
tuberculous etiology. MRI of right wrist and hand (Fig $4,5,6)$ showed thickening of common flexor tendon synovial sheath with peritendinous fluid collection with debris involving the superficial and deep flexor tendons of wrist. The collection showed extension above and below flexor retinaculum with hour glass configuration. There were small areas of erosion and marrow edema involving the articular surface of radiocarpal and distal radioulnar joint with minimal joint effusion suggestive of early arthritis of wrist. With contrast, the thickened synovium and peritendinous soft tissue showed enhancement. Nerve Conduction Study showed decreased Sensory Nerve Action Potential (SNAP) for median and ulnar nerves, decreased Compound Motor Action Potential (CMAP) for median nerve and decreased Nerve Conduction Velocity (NCV) for median and ulnar nerves suggesting entrapment neuropathy of both median and ulnar nerve with sparing of the radial nerve.

FNAC of the swelling was done which showed clusters of histiocytes and few clusters of epitheloid cells in the background of lymphocytes with no caseous necrosis suggestive of granulomatous lesion probably tuberculous origin.

The patient was planned for excision biopsy of the swelling. Under supraclavicular block, with tourniquet control, $8 \mathrm{~cm}$ long incision was made over volar aspect of wrist medial to palmaris longus tendon extending along the ulnar aspect of thenar eminence upto the proximal palmar crease. Skin and subcutaneous tissue was incised, palmaris longus tendon retracted, medial nerve was found to be stretched underneath the flexor retinaculum (Fig 7). Carpal tunnel decompression was done by dividing the flexor retinaculum (Fig. 8) and the stretched median nerve was released. Diffuse swelling arising from the common sheath of flexor tendons of wrist and extending to both the radial and ulnar bursa was exposed (Fig. 9, 10). The cyst got ruptured with the discharge of yellow gelatinous fluid as well as multiple melon seeds or rice bodies (Fig. 11). Debulking tenosynovectomy of the tendon sheaths was done and individual tendons were released from adhesions. The excised specimen was sent for gross and microscopic examination. Wound closure was done after tourniquet release and perfect haemostasis. Below elbow slab was applied.

The post-operative period was uneventful. Suture removal was done on $12^{\text {th }}$ post-operative day (Fig. 12) and active and passive mobilization of the wrist was started. The histopathological examination showed epitheloid granuloma with Langhans type of giant cell with central caseous necrosis suggestive of tuberculous etiology (Fig. 12, 13, 14). Gram staining and ZiehlNeelsen staining of the specimen was found to be negative. Under RNTCP (Revised National Control Programme), the patient was started on Category I antituberculous treatment with initial two months of intensive phase followed by continuation phase for 4 months thrice a week. On completion of ATT, the patient had good functional recovery of right hand without any recurrence on follow-up. (Fig. 15)

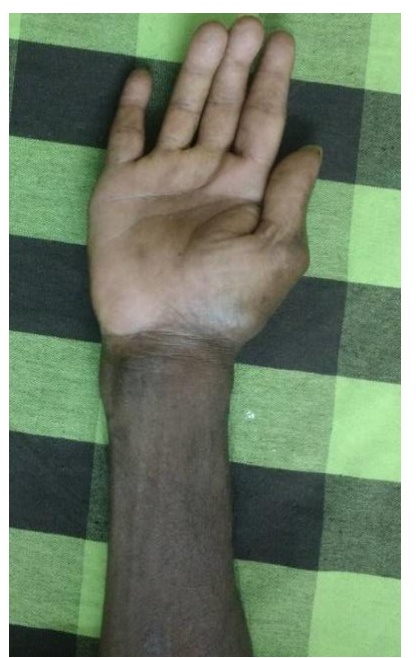

Fig. 1: Pre op clinical picture

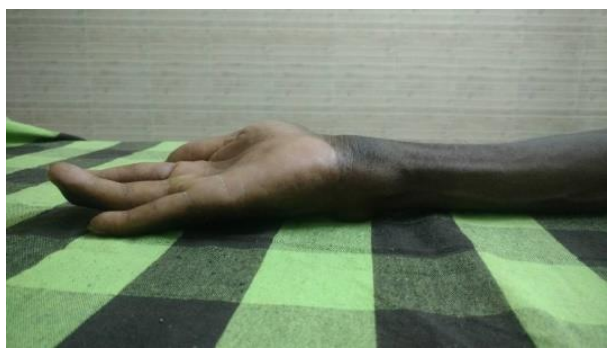

Fig 2: pre op clinical picture

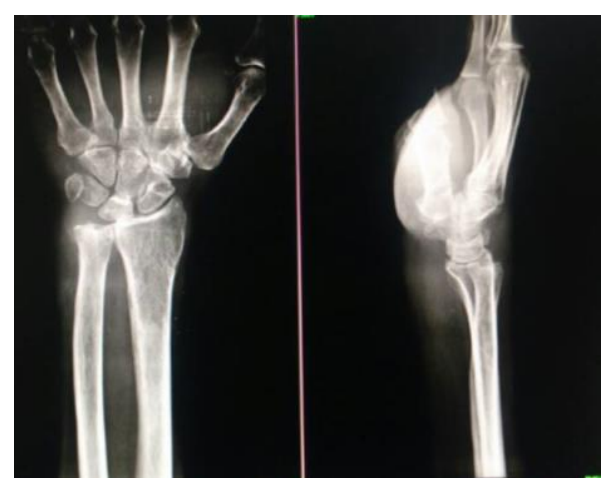

Fig. 3: $X$ ray of right wrist AP and lateral view 


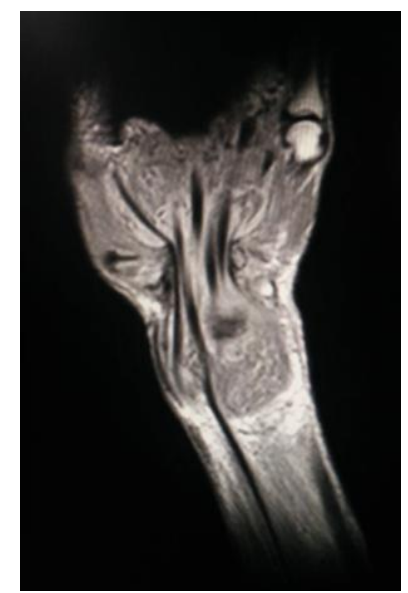

Fig. 4: MRI of the wrist and hand showing hour glass extension of flexor tendon tenosynovitis

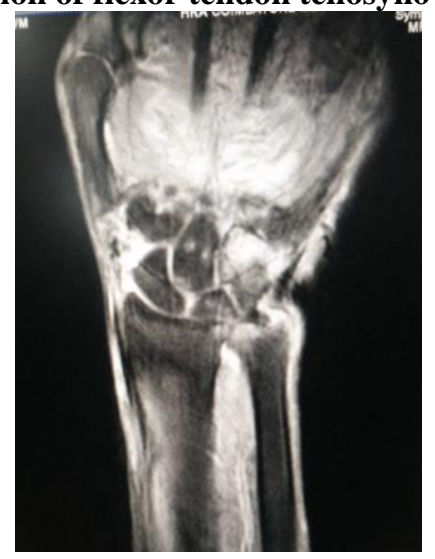

Fig. 5: MRI of the wrist showing small areas of erosion and marrow edema involving the articular surface of radiocarpal and distal radioulnar joint with minimal joint effusion

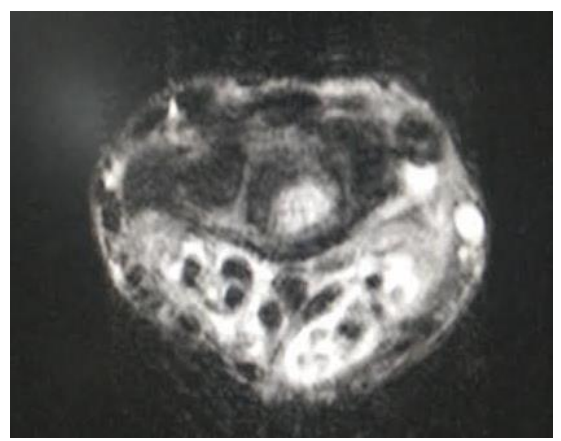

Fig. 6: MRI of the wrist axial section showing thickening of the tenosynovium with peritendinous fluid collection

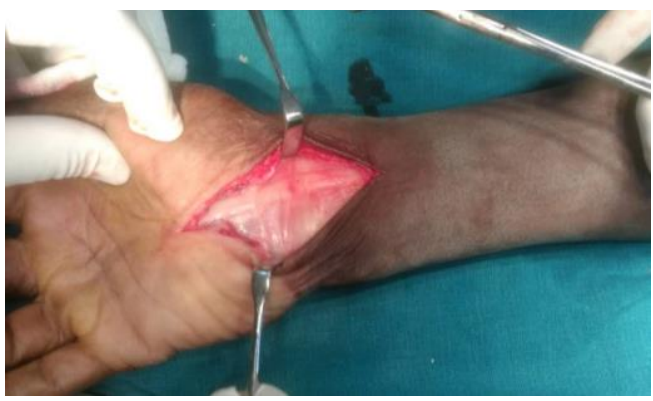

Fig. 7: Intra op picture showing skin incision with the the underlying swelling exposed

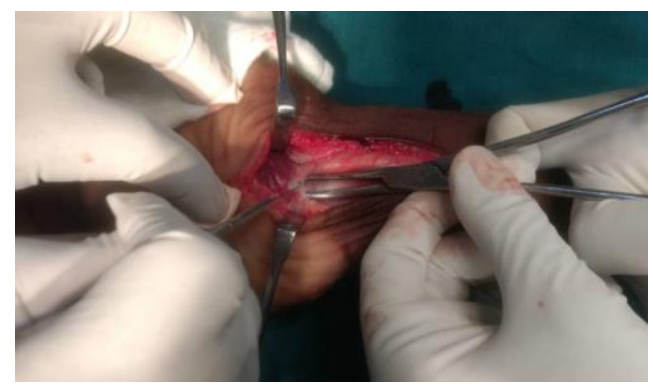

Fig. 8: Intra op picture showing carpal tunnel decompression done by dividing the flexor retnaculum

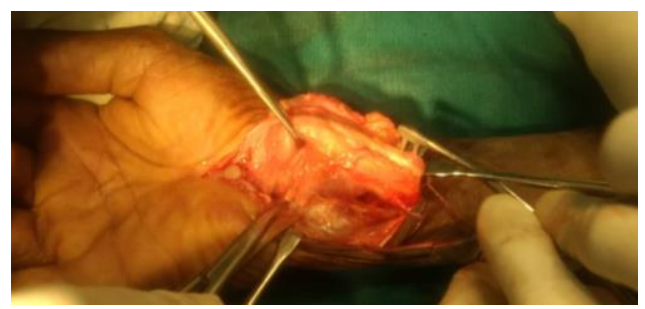

Fig. 9: Intra op picture showing cystic swelling of the common flexor tendon proximal to flexor retinaculum

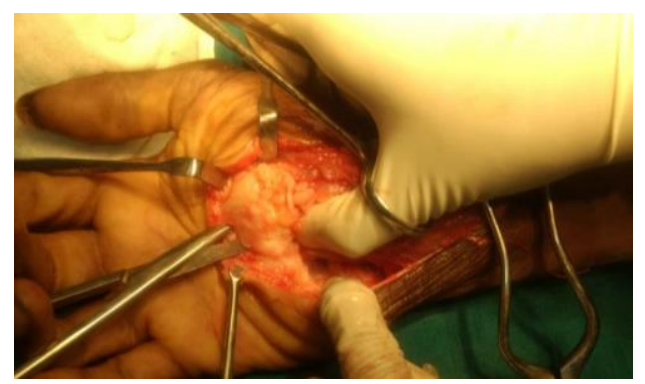

Fig. 10: Intra op picture showing cystic swelling of the common flexor tendon distal to flexor retinaculum 


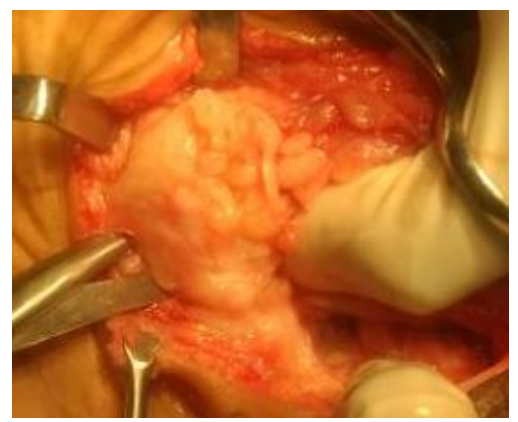

Fig. 11: Intra op picture showing ruptured ganglion with melon seed bodies exposed

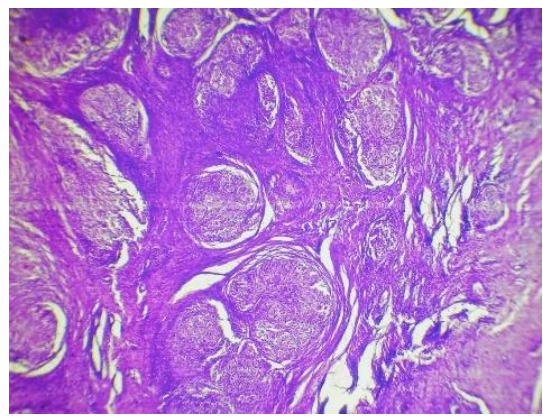

Fig. 12: Histopathological picture with eosin/haematoxylin stain (100X magnification) showing multiple granuloma

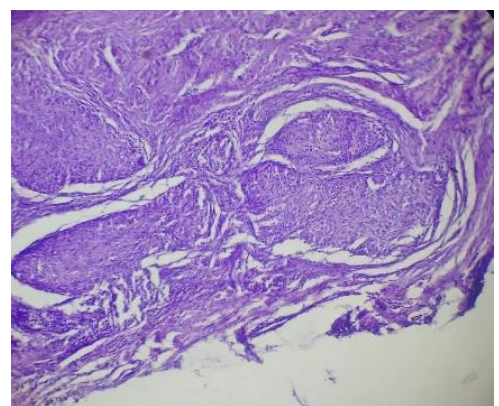

Fig. 13: Histopathological picture with eosin/haematoxylin stain (100x magnification) showing granuloma with langhans giant cell, histiocytes in a background of lymphocytes

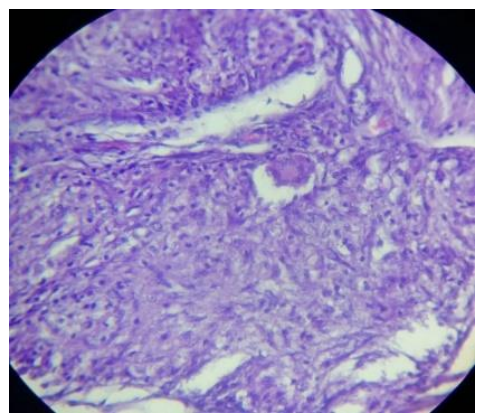

Fig. 14: Histopathological picture with eosin/haaematoxylin stain (400x magnification) showing Langhans giant cell
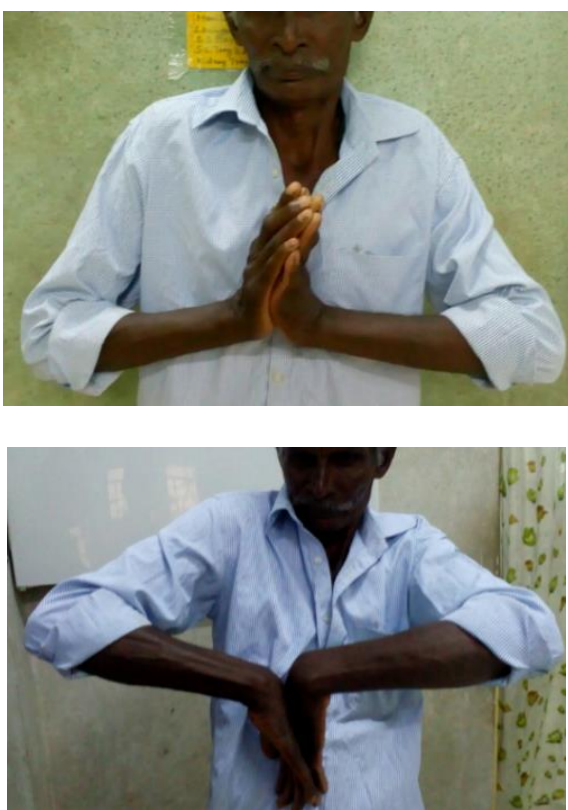

Fig. 15: Post op clinical picture showing ROM

\section{Discussion}

Tuberculosis is globally a major health problem of this century. This is more relevant today because of the prevalence of immunosuppression following HIV/AIDS due to which it has again become an epidemic in many parts of the developing world. Tuberculosis was one of the top ten causes of death worldwide im 2015 ranking above HIV/AIDS among the infectious diseases. According to the "Global Tuberculosis Report 2016", the number of new tuberculosis cases was 10.4 million of which 5.9 million were men, 3.5 million women and 1 million children. Overall $90 \%$ of cases were adults, $10 \%$ children and the male to female ratio was $1.6: 1$ and there were 1.4 million $\mathrm{Tb}$ related deaths in 2015 . The estimated tuberculosis burden of India as of 2015 is as follows: Incidence (includes HIV $+\mathrm{Tb}$ ) $=217$ per one lakh population, Incidence of HIV $+\mathrm{Tb}$ only $=8.6 \mathrm{per}$ one lakh.

The most commonly involved extrapulmonary sites are CNS, lymph nodes, genitourinary tract and musculoskeletal system including bursa, tendons which comprises $1.3 \%$ of all cases having tuberculosis. ${ }^{2}$ The tuberculous tenosynovitis is a rare form of extrapulmonary tuberculosis. The synovial / bursal sheath gets infected by direct haematogenous route from a primary focus in the lung or by direct inoculation from the pre-existing bone and joint disease. It is more commonly seen in upperlimb than lowerlimb. In the upper limb it more frequently involves flexor aspect of the wrist and hand of the dominant limb than the extensor side. ${ }^{3}$

Tuberculous tenosynovitis affecting common sheath of the forearm flexor tendons, extending above and below the flexor retinaculum is classically called "Compound Palmar Ganglion". Pathologically both the parietal and visceral tenosynovium is replaced by 
tuberculous granulations. As the infection progresses, the tendon may fray and rupture spontaneously and secondary involvement of wrist joint may also occur ${ }^{4}$. Both the radial and ulnar bursae are commonly affected in compound palmar ganglion. The radial bursa arises $2.5 \mathrm{~cm}$ proximal to the flexor retinaculum as the synovial sheath of the flexor policies longus tendon and passes beneath the carpal tunnel and continues as digital sheath of the thumb. Similarly, ulnar bursa is the synovial sheath arising from the tendon of the distal phalanx of the little finger and extends proximally to envelope the superficial and deep tendons of the middle, ring and little fingers. There is communication between radial bursa and ulnar bursa in $80 \%$ of cases, which accounts for the horse shoe shaped tenosynovitis. ${ }^{5}$

Onset is characteristically insidious and progression is slow. Pain is usually not significant but once the median nerve compression occurs, then tingling, paresthesia of the lateral three and half fingers occur, more during night, that signify onset of carpal tunnel syndrome..$^{6-8}$ Ho Jung Kang et al. in their study involving twelve patients of carpal tunnel syndrome due to space occupying lesion in the wrist had concluded that tuberculous tenosynovitis was the cause in three cases and ganglion in one case. ${ }^{9}$

Blood investigations are usually normal except for the raised ESR and CRP and positive tuberculin test. ${ }^{10}$ $\mathrm{X}$ ray chest may show evidence of primary focus in the lung but in our case $X$ ray chest was normal. Nerve conduction study may reveal slow conduction velocity initially as a result of myelin sheath damage caused by nerve compression. Later, reduction of motor or sensory action potential amplitude occurs due to axonal loss resulting from sustained or severe compression. The affected muscles innervated by median nerve show either active denervation (e.g. fibrillation and fasciculation potentials) or chronic changes due to denervation followed by subsequent renervation as revealed by Electromyography. ${ }^{11}$ In our case Nerve Conduction Study showed features suggesting entrapment neuropathy of both median and ulnar nerve that is rarely seen in a case of compound palmar ganglion. EMG study was not done in our case.

Sourav Talukder et al., ${ }^{11}$ in their detailed study, have correlated postoperative histopathological findings and the radiological features of tuberculous tenosynovitis by using USG, power Doppler study and contrast MRI. B-mode ultrasonogram is used to visualize proximal and distal extent of the lesion around the flexor tendons. MRI is the best imaging modality and shows thickened synovium and tenosynovium along with fluid component, that is hypotense on T1W1 and hyperintense on $\mathrm{T} 2 \mathrm{~W} 1$. The rim enhancement of the lesion is seen following intravenous post-contrast studies. In contrast to acute suppurative infection, tuberculous tenosynovitis has less fluid collection. On $\mathrm{T} 2$ weighted sequences, hypointense focus, hypointense synovia together with central erosion and abscess with surrounding contrast distinguishes tuberculosis from other kinds of inflammatory arthritis..$^{12}$

The first detailed study of tuberculous tenosynovitis along with three classical histopathological stages was published by Kanavel in 1923. ${ }^{13}$ The three factors determining the stages are the duration of disease, patient resistance and bacterial virulence. In first stage, there is inflammation followed by obliteration and fibrosis of the tendon sheaths. The caseous necrosis lead to formation of fibrinous tubercles or masses, which are seen as rice body/melon seed body in the second stage. The extensive caseation along with rupture of tendon sheath leads to third stage. Kanavel, in his study, has reported 10 cases of tendon rupture among 21 cases. Our case represents the second stage of tuberculous tenosynovitis. Woon et $\mathrm{al}^{14}$ reported that findings of rice body, millet or melon seed body intra-operatively are highly indicative of tuberculous tenosynovitis. ${ }^{14}$

The wide range of differential diagnosis and poor clinical awareness often leads to delay in arriving at clinical diagnosis and subsequent treatment, leading to complications. Differential diagnosis of compound palmar ganglion includes inflammatory arthritis (rheumatoid arthritis, gouty arthritis), sarcoidosis, infective (pyogenic infections, foreign body tenosynovitis, fungal granuloma) and other lesions of the tendon sheath (pigmented villonodular synovitis, giant cell tumour, amyloidosis and synovial chondromatosis). ${ }^{2,3,15,16}$ The diagnosis can be confirmed by open excision biopsy with histopathological examination and mycobaterial culture of the specimen. Lertsrisatit et al. ${ }^{17}$ reported the sensitivity of synovial fluid AFB staining (32\%), culture (80\%), histology(65\%), PCR(63\%) and concluded that PCR can be used as an early diagnostic tool ${ }^{17}$. In our case provisional diagnosis of tuberculosis was made initially by FNAC and was subsequently confirmed by histopathology of the biopsy specimen.

The other series of case reports by Arun Kumar et al., ${ }^{18}$ Seiichi Higuchi et al. ${ }^{2}$, Khurshid Ahmad Baht et al., ${ }^{19}$ Sarkan Bayram et al., ${ }^{20}$ Michel N' guessen Anoumou et al. ${ }^{21}$ showed no radiological evidence of arthritic changes of radiocarpal and distal radio-ulnar joint and involvement of both median and ulnar nerve as in our study. In none of the above case reports, involvement of the nerve was assessed by nerve conduction study.

Tuberculous tenosynovitis should always be ruled out giving steroid injection. Interfering with the disease process before it involves the underlying bones is the main goal of treatment. The treatment of tuberculous tenosynovitis is typically medical (anti tuberculosis therapy) based on standard protocols. Primary surgical treatment should be recommended only in stage two lesions and tendon tears. Benchakroum et al. ${ }^{22}$ concluded that surgery should be 
performed not only in case of complications (abscess, neurological) but also when the medical management fails. $^{22}$

\section{Conclusion}

The tuberculous origin of the chronic flexor tenosynovitis of the wrist should be kept in mind especially in developing countries. The presence of rice body or melon seed body alone does not confirm tuberculous origin as other differential diagnosis should be ruled out. The diagnosis should be confirmed by excision biopsy. The delay between the presentation, diagnosis and management should be minimized to provide a good functional outcome as well as to prevent complications and recurrence of the disease.

\section{References}

1. World Health Organisation, Global Tuberculosis report 2016 (WHO/HTM/TB/2016.13)P - 5.

2. Seiichi Higuchi, Shinichi Ishihara, Hiroyuki Kobayashi and Taidoh Arai: A mass lesion of the wrist: A rare manifestation of tuberculosis. [DOI:10.2169/internalmedicine.47.0495]

3. Pei-Hung Shen, Cheng-Mien Chu, Gua-Shu Huang, Shing-Sheng Wu and Chain-Her Lee tuberculous tenosynovitis of the flexor tendons of the wrist and hand: J Med Sci 2002;22[5]:227-30.

4. Tuli SM. Tuberculosis of the skeletal system. Jaypee, Delhi, 2004

5. Pimm LH, Waugh W. Tuberculous tenosynovitis. J Bone Joint Surg Br. 1957;39- B(1):91-101.

6. Mamoon Rashid, Saad Ur Rehman Sarwar, Ehtesham Ehtesham, Muhammad Zia Ul Islam, Kokab Shah: tubercular tenosynovitis: a cause of carpal tunnel syndrome.JPMA 56:116;2006.

7. A.Gillies tuberculous tenosynovitis of the palmer synovial bursa: compound palmar ganglion. JBJS 193. www.jbjs.org/cgi/reprint13/1/156.

8. Arvind G Kulkarni, Vidyanand V Prabhu. Tuberculosis presenting as carpal tunnel syndrome: a case report with review of literature. http//www.bhj.org/journal/20024401-jan/case-102.htm.

9. Kang HJ, Jung SH, Yoon HK, Hahn SB, Kim SJ. Carpal tunnel syndrome caused by space occupying lesions. Yonsei medical journal. 2009 Apr 30;50(2):257-61.

10. Aboudola S, Sienko A, Carey RB, Johnson S. Tuberculous tenosynovitis. Human Pathology 35: 10441046, 2004.

11. Talukder S, Bandyopadhay A, Biswas S, Chakraborty S, Chakrabarti S. Imaging of Compound Palmar Ganglion with Pathologic Correlation. S Afr J Rad. 2014;18(1);Art. \#654, 4 pages

12. Hsu CY, Lu HC, Shih TT. Tuberculous infection of the wrist: MRI features. Am J Roentgenol. 2004;183(3):6238.

13. Kanavel AB. Tuberculous tenosynovitis of the hand: a report of fourteen cases of tuberculous tenosynovitis. Surg Gynecol Obstet 37:635-47,1923.

14. Woon C.Y., Phoon E.S., Lee J.Y., Puhaindran M.E., Peng Y.P., Teoh L.C. Rice bodies, millet seeds, and melon seeds in tuberculous tenosynovitis of the hand and wrist. A

15. nn. Plast. Surg. 2011;66:610-7.

16. Ergun T., Lakadamyali H., Aydin O. Multiple rice body formation accompanying the chronic nonspecific tenosynovitis of flexor tendons of the wrist. Radiat. Med. 2008;26:545-8.

17. M Tyllianakis, G Kasimatis, S Athanaselis, $M$ Melachrinou: Rice body formation and tenosynovitis of the wrist: a case report. Journal of orthopaedic surgery 2006; 14 [2]:208-11.

18. Lertsrisatit P, Nantiruj K, Totemchokchyakarn K, Janwityanujit S.Extraspinal tuberculous arthritis in HIV era. Clin Rheumatol 26:319-321, 2007.

19. Arun Kumar K, Kanthimathi B, Krishnamurthy CS, Sujai S. Compound palmar ganglion: A tubercular manifestation of flexor tenosynovitis of the wrist. International Journal of Case Reports and Images 2012;3(2):2831.

20. Bhat KA, Gani MH, Singh O, Khan Ad. Tubercular compound palmar ganglion causing carpal tunnel syndrome. Saudi J Sports Med 2015;15:288-90.

21. Bayram S, Erşen A, Altan M, Durmaz H. Tuberculosis tenosynovitis with multiple rice bodies of the flexor tendons in the wrist: A case report. International Journal of Surgery Case Reports. 2016;27:129-32. doi:10.1016/j.ijscr.2016.08.021.

22. Anoumou MN, Kouame M, Daix T, Yepie A (2014) Tuberculosis tenosynovitis of the flexor tendons in the wrist: a case report. Acta Orthop Traumatol Turc 48(6):690-2.

23. Benchakroun M, El Bardouni A, Zaddoug O, Kharmaz M, El Yaacoubi M, Ouadghiri M, et al. Tuberculosis of the wrist. Symptoms and outcome in eleven cases. [Article in French] Rev Chir Orthop Reparatrice Appar Mot 2004;90:337-45. 